Periodica Polytechnica Civil Engineering, 65(1), pp. 120-133, 2021

\title{
Clustering Elements of Truss Structures for Damage Identification by CBO
}

\author{
Mohammad Hosein Talebpour ${ }^{1 *}$, Younes Goudarzi ${ }^{2}$, Mehrdad Sharifnezhad $^{3}$ \\ ${ }^{1}$ School of Engineering, Damghan University, 36716-41167 Damghan, Iran \\ 2 Faculty of Civil Engineering and Geodesy, Graduate University of Advanced Technology, 7631818356 Kerman, Iran \\ ${ }^{3}$ Department of Engineering, Damghan Branch, Islamic Azad University, 3671639998 Damghan, Iran \\ * Corresponding author, e-mail: m.h.talebpour@du.ac.ir
}

Received: 09 June 2020, Accepted: 04 September 2020, Published online: 16 October 2020

\begin{abstract}
The number of structural elements plays a significant role in detecting damage location and severity; such methods have sometimes failed to provide correct solutions due to the entrapment of damage detection algorithms in the local optimum. To resolve this problem, this study proposed the simultaneous use of mathematical and statistical methods to narrow down the search space. To this end, a two-step damage detection method was proposed. In the first step, the structural elements were initially divided into different clusters using the k-means method. Subsequently, the possibly damaged elements of each cluster were identified. In the second step, the elements selected in the first step were placed in a new set, and a process was applied to identify their respective damage location and severity. Thus, the proposed method reduced the search space as well as the possibility of entrapment in the local optimum. Other advantages of the proposed method include the use of fewer dynamic properties. Accordingly, by narrowing down the search space and the dimensions of the system for governing equations, the proposed method could significantly increase the chance of obtaining favorable results in structures with many elements and those with few vibration modes. A meta-heuristic method, called the colliding bodies optimization (CBO), was used in the proposed damage detection optimization algorithm. The optimization problem was based on the modal strain energy equations. According to the results, the proposed method was able to detect the location and severity of damage, even at its slightest percentage.
\end{abstract}

Keywords

damage identification, modal strain energy, clustering of elements, colliding bodies optimization

\section{Introduction}

Structural elements fail for different reasons including construction errors, unpredicted loads, and environmental factors. If damage remains undetected in early stages, the incurred damage will inevitably spread in the structure and will ultimately make the structure collapse. Therefore, the in-time detection of structural damage and failure is essential to increasing the structural safety and reliability. Hence, damage detection systems play a crucial role in improving the safety and performance of structures in addition to preventing property damage and the loss of life in this regard. To detect damage location and severity in the past, different methods were used such as the x-ray method, ultrasonic method, and impact method. Numerical methods have been implemented by researchers in recent years for detecting damage location and severity. Some of such methods detect the location and severity of damage through optimization algorithms based on the response of the structure to external loads. Extensive research has been conducted in this regard in previous years [1].

Doebling et al. [1] were among the first researchers to conduct a comprehensive study on the numerical methods for damage detection. They found that different structural characteristics exhibited different sensitivities to damage and failure. They also realized that some of the methods, applied to structures with multiple damage locations, failed to produce a correct assessment of the failure location. In their subsequent paper, Doebling et al. [1] applied the modal strain energy based on selecting critical modes of vibration to resolve the problem. They found that selecting the dominant vibration modes based on the maximum modal strain energy would improve the accuracy of detecting a damage location [2]. Other researchers 
have used the structural flexibility matrix to determine not only the location, but also the numerical magnitude of the damage. These methods required empirical data to calculate the flexibility matrix using a limited number of modes obtained from experiments. The location and severity of damage could then be identified based on the flexibility matrix [3-4]. Pandey and Biswas [5] proposed a method based on the comparison of flexibility matrices, obtained for damaged and undamaged structures. By evaluating the variations in each column of the flexibility matrix, a damage matrix was defined and used to study the damage detection problem. Sohn and Law [6] used the Ritz vectors for damage detection. The Ritz vectors were calculated by obtaining the product of the flexibility matrix and the dynamic-load-dependent vector. Shi et al. [7] proposed a method based on the modal strain energy for detecting the damage location in structures. They based their method on comparing the respective modal strain energy levels in structural elements before and after the damage. Guan and Karbhari [8] improved the modal-strain-energy damage detection method by proposing a damage index which could be calculated from only the modal displacement and modal rotation through the penalty method. Yang [9] developed a damage index to detect failure in plates based on the modal strain energy of astructure. Presenting the structural damage problem as an optimization problem, Chou and Ghaboussi [10] employed a meta-heuristic GA algorithm to detect structural failure location and severity. Introducing a multi-objective problem, Perera et al. [11] utilized a combination of the particle swarm optimization (PSO) and genetic algorithms to evaluate structural damage. Simulating the structural damage problem as a constrained optimization problem, Wang et al. [12] used the GA to study damage detection in a roof truss. Seyedpoor [13] combined the meta-heuristic PSO algorithm with a two-step method based on the modal strain energy method to examine the structural damage detection. Kaveh and Maniat [14-15] used novel MCSS optimization methods for damage detection in truss structures and beams. The objective function of the optimization problem was defined using the penalty function based on the dynamic structural responses and the effect of noises on data. The results of optimization by the PSO algorithm were compared with those of the MCSS algorithm. Different numerical examples confirmed the capability of the MCSS algorithm in detecting the location and severity of damage despite noises in the dynamic responses. Kaveh and Mahdavi [16] also used meta-heuristic algorithms to detect structural damage. Kaveh and Zolghadr [17] proposed a new guided modal strain energy-based approach for structural damage identification. The damage identification problem was formulated as an inverse optimization problem with a damage sensitivity objective function based on the flexibility matrix. Using a criterion based on the modal strain energy, the search space was reduced by separating structural members prone to damages. To evaluate the proposed method, various noise levels were considered up to $1.5 \%$ and used for solving the optimization problem by the TOW algorithm. Despite the few vibration modes, the results of numerical examples indicated the capability of the proposed method. Kaveh and Dadras [18] solved the damage identification problem by the ETEO algorithm as the extended version of the TEO algorithm. The damage identification problem was defined as an inverse optimization problem. To evaluate the performance of the proposed algorithm, several numerical examples were solved under different damage scenarios with and without noises. The results indicated the capability of the proposed algorithm in detecting damages in structural members with an error of less than $1 \%$. Kaveh and Zolghadr [19-20] proposed a new cylindrical parthenogenesis algorithm (CPA) for damage detection based on the modal strain energy. The damage detection problem was defined as an inverse optimization problem, and the structural flexibility matrix was used as the objective function. The results indicated the superior capability of the CPA in comparison with the PSO, RO and FA algorithms. Using the few first structural modes in the numerical examples, this algorithm correctly detected the location and severity of damage.

Evaluations show that the damage extent can be determined with a certain error with increasing the search space and number of unknowns. Therefore, the search space can be reduced by a strategy to avoid this issue. Through a new idea of clustering structural elements, it was possible to narrow down the search space. In the proposed method, an initial evaluation of the relevant structural parameters was performed on a system with reduced degrees of freedom to avoid numerical problems. To this end, the structural elements were initially clustered through the $k$-means method. Then, the damage detection problem was simulated for each cluster as an unconstrained optimization problem (i.e. a system of equations with fewer unknowns). In the next step, the CBO meta-heuristic algorithm was applied to each cluster to solve the optimization problem. Upon comparing the respective stresses obtained for the 
elements in each cluster, those elements with higher probabilities of failure were selected as suspected damage elements. The other elements were eliminated from the cluster. Upon examining every cluster in this manner, the suspected damage elements were classified in a new cluster, and subsequently used for solving the damage problem. Three examples were presented to demonstrate the efficiency of the proposed method (in terms of narrowing down the search space and obtaining favorable answers) using only a few vibration modes of the analyzed structure.

\section{Damage identification}

\subsection{Modal strain energy}

The strain energy of a structure expresses the effects of the external factors on that structure, as explained below.

$U=\frac{1}{2}\{\Phi\}^{T}[K]\{\Phi\}$

In Eq. (1), $U$ represents the total modal strain energy of the system when $\{\boldsymbol{\Phi}\}$ and $[K]$ express the vibration modes and stiffness matrix of the structure, respectively [7].

A damage suspected in structural elements would lead to changes in structural characteristics including structural stiffness and structural frequency. Such variations would, in turn, result in the creation of a new strain energy level in the damaged structure. The total strain energy of the system is expressed as

$\Delta U=U^{h}-U^{d}$.

In Eq. (2), $\Delta U, U^{h}$, and $U^{d}$ represent the total strain energy of the system, the strain energy of the undamaged structure, and the strain energy of the damaged structure, respectively. Meanwhile, structural stiffness is the main cause of variations in structural strain energy, while variations of other structural parameters are merely the results of the changes imposed on the stiffness matrix. Thus, the strain energy of the damaged structure can be properly studied by applying changes to its stiffness matrix. The stiffness matrix of the damaged structure is expressed [21].

$[K]^{d}=[K]^{h}-[\delta K]$

In Eq. (3), $[K]^{d},[K]^{h}$, and $[\delta K]_{j}$ are the stiffness matrix of the damaged structure, the stiffness matrix of the undamaged structure, and the variations of the stiffness matrix, respectively. By applying Eq. (3) to each element, we can obtain the stiffness matrix for that element as

$[\delta k]_{j}=\alpha_{j}[k]_{j}^{h} \quad j=1, \ldots, n e$.
In Eq. (4), $\alpha_{j},[K]_{j}^{h}$, and $[\delta K]_{j}$ represent the extent of damage, the stiffness, and the stiffness variations in the $j$-th element of the structure, respectively. Also, ne denotes the number of the structural elements. By placing Eq. (4) in Eq. (3) for each element, we can obtain the stiffness matrix for a damaged element as

$[k]_{j}^{d}=[k]_{j}^{h}-\alpha_{j}[k]_{j}^{h} \quad j=1, \ldots, n e$.

In the above equation, $[K]_{j}^{d}$ is the stiffness matrix of the $j$ th element in the damaged structure. By expressing the total strain energy of the structure (Eq. (1)) as the sum of the energies of the individual elements, we can obtain the total structural energy variations for the damaged structure (based on Eqs. (2) and (5)) as [22].

$$
\begin{aligned}
\Delta U & =\left(\sum_{j=1}^{n e} \frac{1}{2}\left\{\phi_{j i}^{h}\right\}^{T}\left[k_{j}^{h}\right]\left\{\phi_{j i}^{h}\right\}\right) \\
& -\left(\sum_{j=1}^{n e} \frac{1}{2}\left\{\phi_{j i}^{d}\right\}\left([k]_{j}^{h}-\alpha_{j}[k]_{j}^{h}\right)\left\{\phi_{j i}^{d}\right\}\right)
\end{aligned}
$$

In Eq. (6), $n q$ represents the number of the existing modes of vibration when $\{\boldsymbol{\phi}\}_{j i}^{h}$ and $\{\boldsymbol{\phi}\}_{j i}^{d}$ are the modal shape vectors of the $j$ th element of the ith mode obtained for the undamaged and damaged structures, respectively. Thus, Eq. (6) can be expressed as a system for linear equations comprising $n e$ unknowns and $n q$ equations. By applying the second term on the right-hand side of Eq. (6) to the structure as a whole, Eq. (7) is obtained

$$
\begin{aligned}
\Delta U & =\left(\sum_{j=1}^{n e} \frac{1}{2}\left\{\phi_{j i}^{h}\right\}^{T}\left[k_{j}^{h}\right]\left\{\phi_{j i}^{h}\right\}\right)_{i=1, \ldots, n q .} \\
& -\left(\frac{1}{2}\left\{\phi_{i}^{d}\right\}^{T}\left[K^{d}\right]\left\{\phi_{i}^{d}\right\}\right)
\end{aligned}
$$

Hence, the stiffness matrix for the damaged structure can be expressed as

$[K]^{d}=[M] \lambda_{i}^{d} \quad i=1, \ldots, n q$.

In Eq. (8), $[M]$ is the mass matrix and $\lambda_{i}^{d}$ is the $i$ th natural frequency of the damaged structure. Considering the orthogonality of vibration modes, we can equate Eqs. (6) and (7) (using Eq. (8)) to obtain Eq. (9).

$$
\sum_{j=1}^{n} \alpha_{j}\left\{\phi_{j i}^{d}\right\}^{T}\left[k_{j}^{h}\right]\left\{\phi_{j i}^{d}\right\}=\sum_{j=1}^{n}\left\{\phi_{j i}^{d}\right\}^{T}\left[k_{j}^{h}\right]\left\{\phi_{j i}^{d}\right\}-\lambda_{i}^{d}
$$

As the above equation expresses a system for linear equations with ne unknowns (i.e. the number of the struc- 
tural elements) and $n q$ equations (i.e. the number of vibration modes), we can write Eq. (9) in a matrix form to obtain Eq. (10) as.

$$
\begin{aligned}
{[S]\{\alpha\}=} & \{\Delta \boldsymbol{R}\} \Rightarrow \\
& {\left[\begin{array}{ccc}
s_{11} & \ldots & s_{n e 1} \\
\vdots & \ddots & \vdots \\
s_{1 n q} & \cdots & s_{n q n e}
\end{array}\right]\left\{\begin{array}{c}
\alpha_{1} \\
\vdots \\
\alpha_{n e}
\end{array}\right\}=\left\{\begin{array}{c}
\Delta \boldsymbol{R}_{1} \\
\vdots \\
\Delta \boldsymbol{R}_{n q}
\end{array}\right\} }
\end{aligned}
$$

In this equation, the individual elements of matrix $[S]$ and variation vector $\{\Delta \boldsymbol{R}\}$ are expressed as follows.

$$
\begin{gathered}
S_{j i}=\left\{\boldsymbol{\phi}_{j i}^{d}\right\}^{T}\left[k_{j}^{h}\right]\left\{\boldsymbol{\phi}_{j i}^{d}\right\} \quad j=1, \ldots, n e \quad i=1, \ldots, n q \\
\Delta \boldsymbol{R}_{i}=\sum_{j=1}^{n e}\left\{\boldsymbol{\phi}_{j i}^{d}\right\}^{T}\left[k_{j}^{h}\right]\left\{\boldsymbol{\phi}_{j i}^{d}\right\}-\lambda_{i}^{d} \quad i=1, \ldots, n q
\end{gathered}
$$

In Eq. (11), $S_{i j}$ is the modal strain energy of the $j$ th element in the $i$ th mode of vibration. In Eq. (12), $\Delta \boldsymbol{R}_{i}$ indicates the response variation in the $i$ th mode, measured experimentally or obtained from the analytical model.

\subsection{Objective function}

Assigning a suitable objective function to the damage detection problem requires implementing statistical identification methods for the structure. These methods are based on inverse solution of the governing structural vibration equations and using the vibration characteristics obtained from the sensors. Thus, the previously known parameters in Eq. (10) can be calculated. Eq. (10) is a system for linear equations with $n q$ equations and $n e$ unknowns. As the number of the measured responses (i.e. the number of equations or the number of vibration modes) in the damage detection problem is smaller than that of the structural unknowns (i.e. structural elements), this system has an infinite number of solutions. Therefore, to find the suitable solution, we may apply statistical methods. The least squares method is an efficient method for solving this system of equations [23-24]. To solve this system of equations through the least squares method, we consider the error vector $\{\varepsilon\}$, expressed as.

$\{\varepsilon\}=\{\Delta \boldsymbol{R}\}-[S]\{\alpha\}$

The error vector can then be defined as Eq. (14).

$\|\varepsilon\|=\|\Delta \boldsymbol{R}-S \alpha\|^{2}$

In most cases, the sum of the squares differences is obtained from the respective measured and estimated values [23-24]. However, due to the mathematical-structural nature assumed in this paper for the function in Eq. (14), the solution of the damage detection problem would require redefining the problem in the form of a linear iteration and an optimization problem. To this end, the obtained analytical model must be evaluated after each iteration. Iteration methods try to improve the relationship between the numerical data and the analytical model (or the experimental results) using the relevant dynamic characteristics of the structure through the objective function. Indeed, if the parameter variations, obtained for successive iterations, are sufficiently small, a good evaluation of the model can be obtained. It can then be used to improve the efficiency of the system. Accordingly, the proposed method was used in this paper to solve the above system for linear equations through an iterative method by redefining the problem as an unconstrained optimization problem. Then, the respective damage severity $\{\boldsymbol{\alpha}\}$ was obtained for each damaged element. Thus, the general form of the damage detection problem can be formulated as.

Find : $\{\alpha\}=\left[\begin{array}{lll}\alpha_{1} & \ldots & \alpha_{n e}\end{array}\right]^{t}$

Minimize $: W(\alpha)=\|S \alpha-\Delta \boldsymbol{R}\|^{2}$

In Eq. (15), $\{\boldsymbol{\alpha}\}$ is the damage variables vector, including the location and severity of the damage, incurred on the structural elements. The damage variables vary within the $0-1$ interval and are defined as reductions in the modulus of elasticity of the elements.

$\alpha_{j}=1-\frac{\left(E_{d}\right)_{j}}{E_{h}} \quad j=1, \ldots, n e$

In the above equation, $E_{d}$ and $\left(E_{h}\right)_{j}$ indicate the modulus of elasticity of the undamaged elements and the $j$ th damaged element, respectively.

\subsection{Clustering of elements}

In actual optimization problems, too many iterations are required to obtain the optimum solution due to the many design variables involved. In addition, the iteration technique generates local optimums in certain cases, and the research problem, namely the damage detection optimization problem, is no exception in this regard. Considering the number of dimensions in the governing system of equations, we require an efficient algorithm capable of executing numerous iterations to obtain a suitable solution to the damage detection problem. Thus, it was decided to apply the $k$-means clustering method to the damaged elements for increasing the chance of finding the required damage 
location and severity values. First proposed in 1935, the clustering method classifies the data in order to subdivide the search space into subspaces in smaller sizes [25-26]. By selecting suitable criteria for the existing problem, we can employ this method to classify the relevant variables into different groups. Different clustering methods can be used for different problems. Upon examining the existing methods, the $k$-means method was used for the first time to solve the damage detection problem. Based on an iteration method, the $k$-means clustering method assigns certain points as cluster centers (cluster representative). In the subsequent steps, each cluster was formed by detecting the elements on the shortest distance [27]. The equation used as a criterion in the clustering iteration process is expressed as

$$
J=\sum_{k=1}^{p} \sum_{l=1}^{q}\left\|x_{l}^{k}-m_{k}\right\|^{2} .
$$

In Eq. (18), $m_{k}$ represents the center (representative) of the $k$ th cluster and $x_{l}^{k}$ is the position of the $l$ th element of the $k$ th cluster. Furthermore, $p$ and $q$ are the number of elements and the number of clusters (used in the $k$-means clustering method), respectively. This equation was used to calculate the distance between each element and its cluster center. The iteration process was then operationalized until the suitable value was obtained [26-27]. The k-means clustering method was used - for the first time - in this paper for detecting structural damage. The following process was proposed for classifying the structural elements and applying the clustering algorithm.

Initially, the candidate elements were selected to be distributed among the clusters upon comparing the respective stresses obtained for the undamaged and the damaged elements. Thus, if the stress in an element changed, that element would be a suspected damage element and be consequently selected for clustering. In this way, some of the structural members were excluded from the clustering process. Then, random points were generated as cluster representatives in the required number (equal to that of the required clusters). In our optimization problem, these points were selected from the suspected damage elements based on the stress, developed in each element. The respective stress differences between the suspected damage elements and the corresponding representative elements were then calculated with the desired element being placed in the cluster, at the shortest distance from the respective cluster representative. Upon assigning the elements to the respective clusters, each cluster representative was updated by calculating the relevant mean value among the cluster members. Then, the elements were classified in clusters again based on their difference from the respective cluster representative value (i.e. the mean value calculated for each cluster). This procedure was performed until no change was detected in the value of each cluster. The following step-by-step procedure was proposed for clustering the elements in the damage detection problem.

1. Compare the stresses developed in the respective undamaged and damaged elements and select the suspected damage elements.

2. Select $p$ points as cluster representatives from the suspected damage elements in the structure.

3. Assign each member of the suspected damage elements set to a cluster with the minimum stress difference with its cluster representative (Eq. (18)).

4. Upon updating the mean stress value for each cluster, assign the obtained stress value to the new representative.

5. Continue Steps 3 and 4 until no change is detected in the stress value of each representative.

\subsection{Colliding Bodies Optimization (CBO)}

The CBO algorithm is a meta-heuristic algorithm, developed first by Kaveh and Mahdavi [28] by implementing the laws of physics. This algorithm is based on the one-dimensional collision between bodies (regarded as mass particles). It can be used for finding solutions to the optimization problem. Before colliding with another particle, each particle possesses an initial mass and velocity. Following the collision, each particle separates from others at a specific velocity, travelling from its initial position to a new (secondary) position. The secondary position can have a better (or worse) fitness, compared with the initial position. The procedure for this algorithm is summarized as below [29].

In this algorithm, the number of variables in the search space is equal to that of each cluster. First, each particle is assigned a mass based on its fitness, and the particles are then sorted in a descending order in terms of their fitness. Next, the particles are divided into two groups. The first group contains the fixed (non-moving) particles, while the second group includes the moving particles. The fitness of the moving particles is smaller than that of the fixed particles. The positions of the fixed particles in the search space change once the moving particles have collided with them. The respective velocities of the moving and fixed particles are obtained from the following equations. 


$$
\left\{\begin{array}{l}
V_{i}=0 \quad i=1,2,3, \ldots, \frac{n p}{2} \\
V_{i}=X_{i-\frac{n p}{2}}-X_{i} \quad i=\frac{n p}{2}+1, \frac{n p}{2}+2, \ldots, n p
\end{array}\right.
$$

In Eq. (19), $n p$ is the number of the particles with $X_{i}$ and $V_{i}$ being the position and the velocity of the $i$ th particle, respectively. According to the conservation of momentum principle, the momentum of a system of particles (comprising two particles here) before the impact is equal to the post-impact momentum. Therefore, we can equate the kinetic energies before and after the impact to obtain the respective velocities of the non-moving and the moving particles after the impact $\left(V_{i}^{\prime}\right)$.

$$
\left\{\begin{array}{c}
V_{i}^{\prime}=\frac{\left(m_{i+\frac{n p}{2}}+(\mu)\left(m p_{i+\frac{n p}{2}}\right)\right) V_{i+\frac{n p}{2}}}{m p_{i}+m p_{i+\frac{n}{2}}} \quad i=1,2,3, \ldots, \frac{n p}{2} \\
V_{i}^{\prime}=\frac{\left({ }^{m p_{i}}-(\mu)\left(m p_{i-\frac{n p}{2}}\right)\right) V_{i}}{m p_{i}+m p_{i-\frac{n p}{2}}} \quad i=\frac{n p}{2}+1, \frac{n p}{2}+2, \ldots, n p
\end{array}\right.
$$

In Eq. (20), $m p_{i}$ is the mass of the $i$ th particle, expressed as:

$$
m p_{i}=\frac{\frac{1}{f i t(i)}}{\sum_{k=1}^{n p} \frac{1}{f i t(k)}} \quad i=1,2,3, \ldots ., n p
$$

In Eq. (21), fit(i) denotes the value of the objective function obtained for the $i$ th particle. To improve searching in the search space, the $\mu$ coefficient was defined in Eq. (20) in the form of Eq. (22).

$$
\mu=1-\frac{\text { iter }}{\text { iter }_{\max }}
$$

In Eq. (22), iter is the number of the current iteration and iter $_{\max }$ shows the total number of iterations in the optimization process. Ultimately, the new position of each particle was obtained from its pre-impact velocity as:

$$
X_{i}^{\text {new }}=\left\{\begin{array}{l}
X_{i}+\text { rand } . V_{i}^{\prime} \quad i=1,2,3, \ldots, \frac{n p}{2} \\
X_{i-\frac{n p}{2}}+\text { rand } . V_{i}^{\prime} \quad i=\frac{n p}{2}+1, \ldots, n p
\end{array}\right.
$$

In Eq. (23), rand is a random number belonging to the $0-1$ interval, and $X_{i}^{\text {new }}$ denotes the new position of the $i$ th particle after the impact.

\section{Damage detection based on proposed method}

In the previous sections, a detailed discussion was presented for the evaluation steps required in the proposed clustering method for optimization problems. These steps are necessary for detecting the damage location and severity-through the CBO algorithm-based on the modal strain energy method. Accordingly, the idea proposed for damage identification is as in Fig. 1.

\section{Numerical examples}

To check the efficiency of the proposed method, two structures were examined. In all examples, upon applying the Northridge seismic acceleration, the time history analysis was conducted on the structure. In line with the proposed idea, we clustered the structural elements in three clusters. The conventional methods were also applied (without clustering the elements) to each example in the damage scenario, to compare the respective results. It showed that the proposed method was highly favorable in terms of performance. Accordingly, and for brevity, the comparison results were presented and discussed only in the first example (Damage Scenario I).

\subsection{A 31-bar planar truss}

The studied structure is a truss, shown in Fig. 2. It has 31 ele-ments and 14 nodes. It was assumed that the first 10 vibration frequencies were the only data available for determining the stiffness matrix and other structural char-

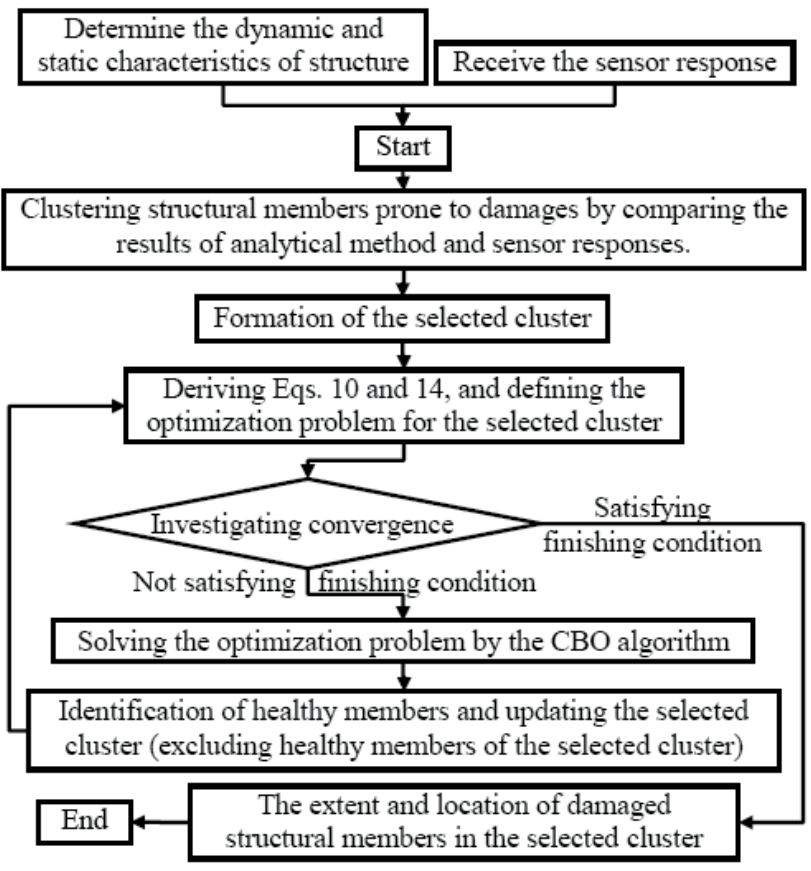

Fig. 1 A Flowchart of the idea proposed for damage identification 


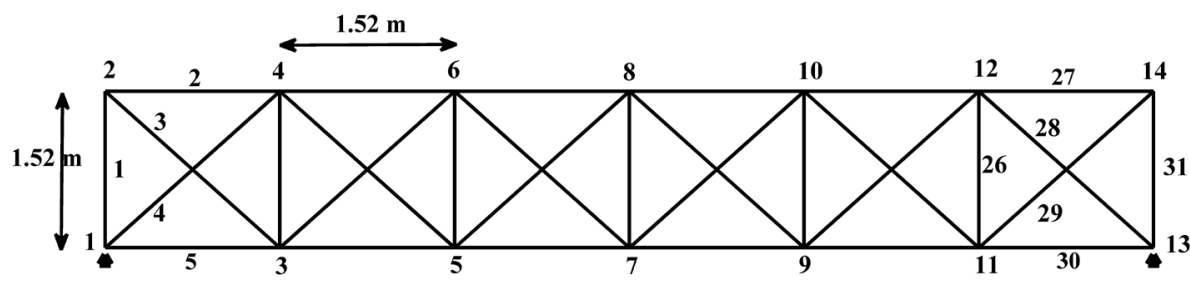

Fig. 2 Planar truss with 31 elements

acteristics of the damaged structure. Note that only the first three frequencies were used here for detecting damage location and severity through the clustering algorithm. However, in the conventional methods (which do not use the clustering algorithm), three cases with three, five, and eight vibration modes were studied. For all elements, $E$ and $\rho$ were assumed to be $70 \mathrm{GPa}$ and $2770 \mathrm{~kg} / \mathrm{m}^{3}$, respectively. The cross-section of all the elements was assumed to be $40 \mathrm{~cm}^{2}$. In this example, three damage scenarios were considered, as listed in Table 1 [30]. The clustering results in Section 2.2 were applied to identify the cluster elements in each scenario (Table 2).

Upon forming the clusters, the location and severity of damage in each cluster for each scenario were determined through the $\mathrm{CBO}$ algorithm. Fig. 3 displays the damage results obtained for the members of the selected clusters in the first case of the damage scenario.

As observed in Fig. 3, the actual damaged elements, i.e. Element 11 and Element 25, are present in the selected cluster. Accordingly, the elements of the selected cluster required for applying the second step of the proposed algorithm are $\{1,2,6,10,11,14,19,25,27\}$.

In the next step, the location and severity of damage in the damaged elements are determined by forming the selected cluster and running the $\mathrm{CBO}$ algorithm. Fig. 4

Table 1 Three different damage scenarios induced in 31 bar planar truss

\begin{tabular}{lcc}
\hline Scenario & Element No & Damage ratio \\
\hline \multirow{2}{*}{ I } & 11 & 0.25 \\
& 25 & 0.15 \\
II & 16 & 0.3 \\
\multirow{2}{*}{ III } & 1 & 0.3 \\
& 2 & 0.2 \\
\hline
\end{tabular}

Table 2 Clustering of the truss elements (31 bar)

\begin{tabular}{|c|c|c|c|}
\hline $\begin{array}{l}\text { Cluster } \\
\text { No. }\end{array}$ & $\begin{array}{l}\text { The members of } \\
\text { cluster Scenario I }\end{array}$ & $\begin{array}{l}\text { The members of } \\
\text { cluster Scenario II }\end{array}$ & $\begin{array}{l}\text { The members of } \\
\text { cluster Scenario III }\end{array}$ \\
\hline 1 & $10,11,13,14,19$ & 31 & $1,2,3,4,6,7$ \\
\hline 2 & $1,2,3,4,5,6,8$ & $20,23,25,26$ & $\begin{array}{c}10,13,14,15,18 \\
19,20,26\end{array}$ \\
\hline 3 & $\begin{array}{c}23,25,27,28,29 \\
30,31\end{array}$ & $\begin{array}{c}8,9,10,12,13 \\
16,17\end{array}$ & $\begin{array}{c}21,23,24,25,28 \\
29,30,31\end{array}$ \\
\hline
\end{tabular}

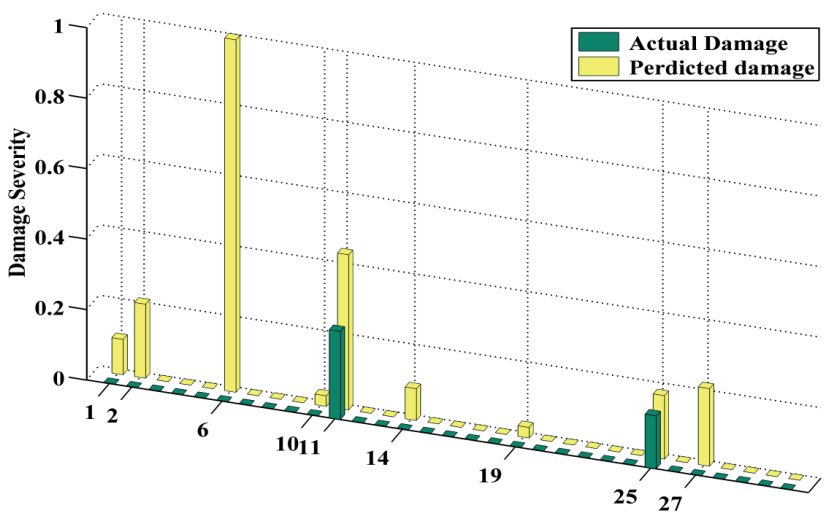

Fig. 3 Suspected damage elements based on clustering-scenario I

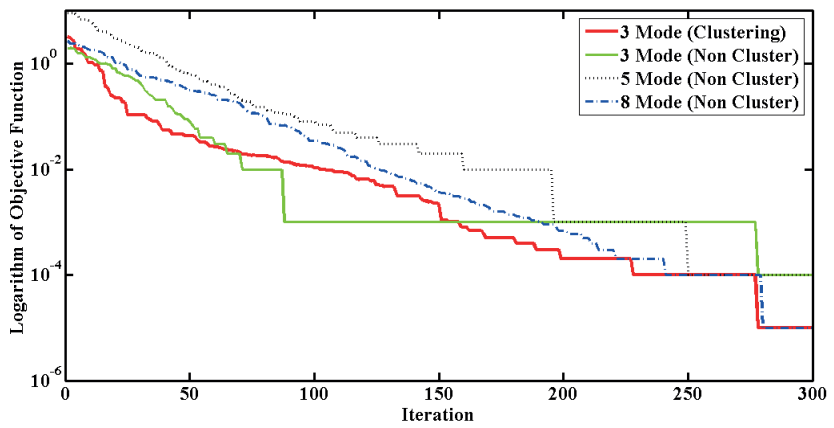

Fig. 4 Convergence of the optimization process to specify the location and severity of damage (31-bar)

reveals the convergence trend of the optimization process, obtained for the selected cluster without implementing the clustering algorithm. As already mentioned, three cases were considered for the conventional process (which did not implement clustering): Case 1 using three vibration modes; Case 2 using five modes; and Case 3 using eight modes. Note that only three structural vibration modes were used where the clustering algorithm was implemented.

As observed in Fig. 4, the convergence trend is far more suitable than that of other cases. The nearest solution resulting from the non-clustering method to that of the proposed clustering method was obtained for the case where eight modes of vibration were assumed. However, the final solutions and convergence trends, obtained from the other non-clustering cases, did not lead to suitable damage detection performances. Note that increasing the number of equations in the damage detection process is highly 
favorable due to the relatively great number of variables involved in this problem. Meanwhile, as the number of equations is equal to the number of vibration modes, applying higher vibration modes would positively affect the optimization process. However, in actual damage detection problems, there is no easy way of obtaining many modes. For this reason, it is important to use methods that can produce suitable solutions using a small number of vibration modes. This goal was achieved in the proposed method as this method was based on only a few modes. In other words, we can apply the proposed damage detection method using only a few modes (much fewer than those used in the conventional methods) to obtain results equal or better than those obtained from the conventional methods. The diagrams in Fig. 4 demonstrate the above concepts as well as the fact that the proposed method is suitable in terms of both efficiency and performance. Note that the vertical axis in these diagrams represents the logarithmic values of the objective function. This facilitates the way variations in the course of convergence are demonstrated, particularly during the final iterations. In addition, it can better

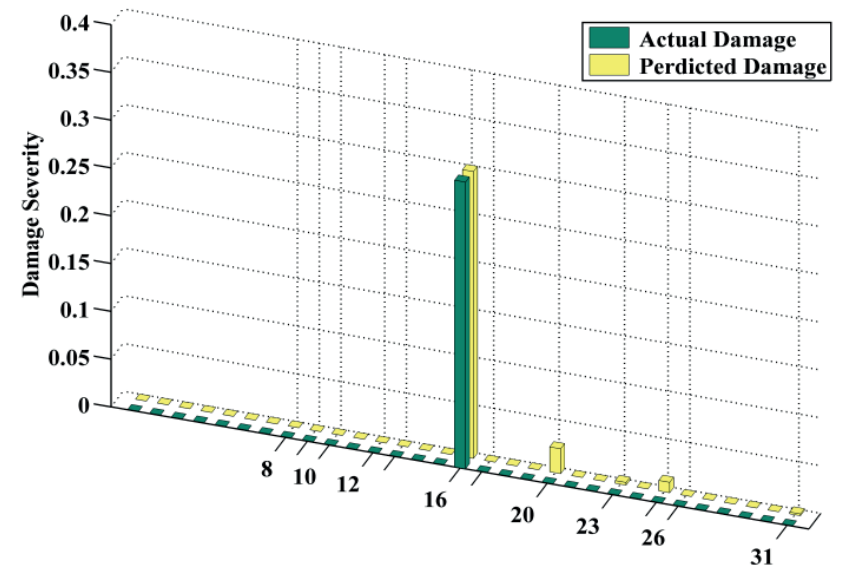

Scenario II compare the convergence trends of the proposed and the conventional methods. Fig. 5 indicates the damage location and severity results obtained in two cases (with and without clustering) through damage Scenario I for the damaged structural elements during the last step of the proposed algorithm. Fig. 5 depicts the final results obtained from the proposed clustering method. As observed, the results exactly matched the actual outcomes. Conversely, none of the results obtained from the three conventional methods

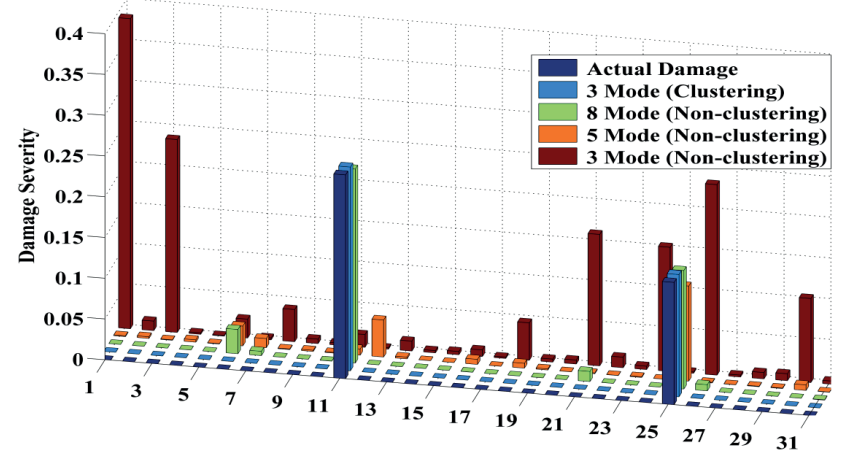

Fig. 5 Identified damage elements-scenario I (31-bar)

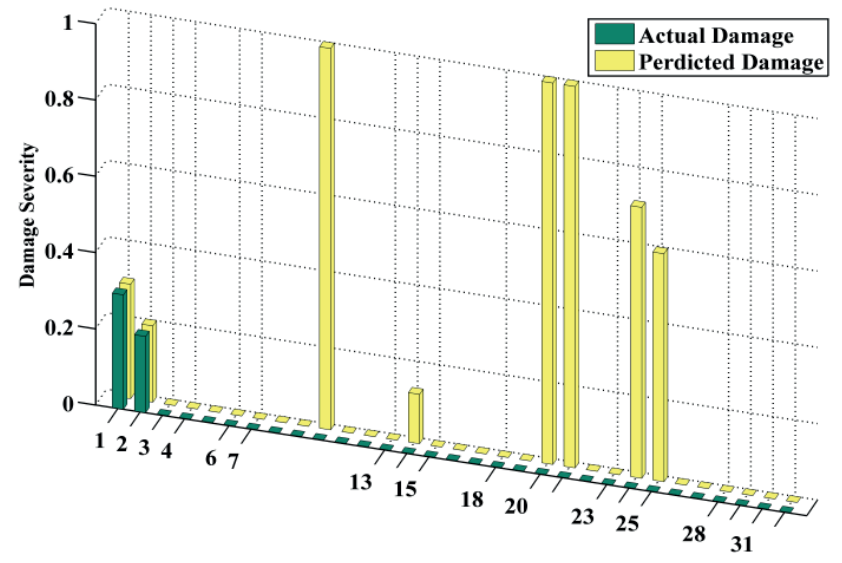

Scenario III

Fig. 6 Suspected damage elements based on clustering (31-bar)
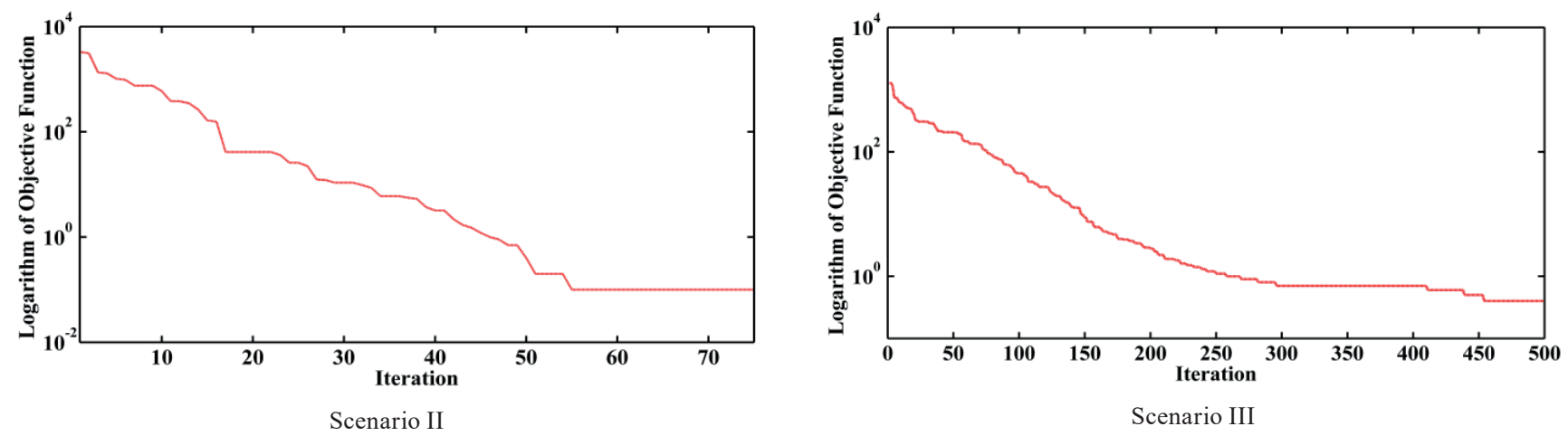

Fig. 7 Convergence of the optimization process to specify the location and severity of damage (31-bar) 


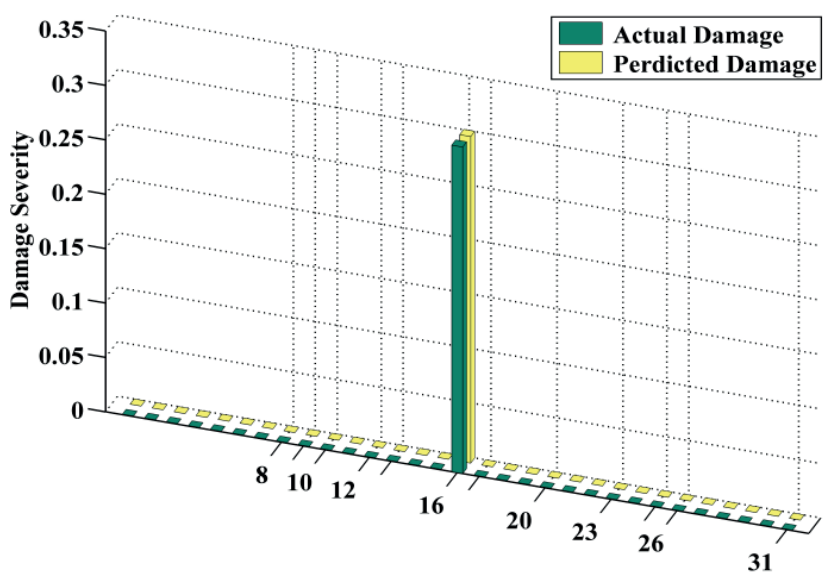

Scenario II

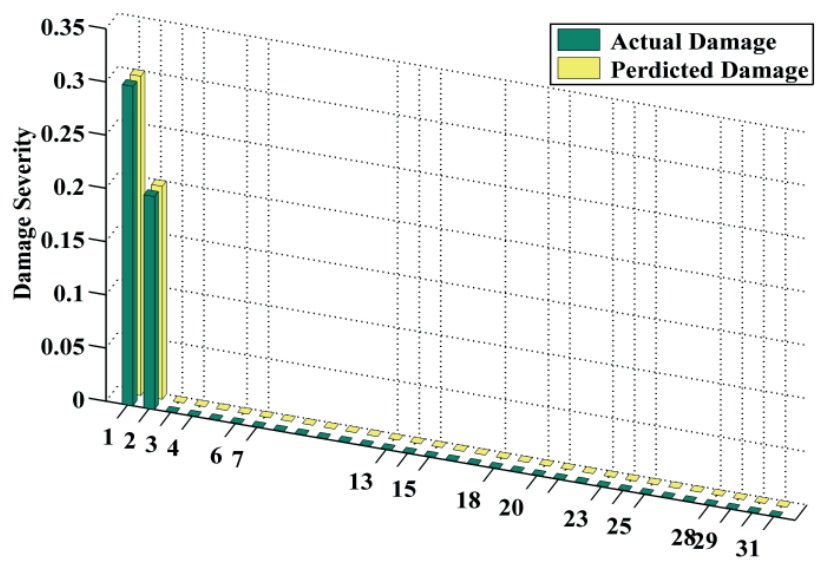

Scenario III

Fig. 8 The damage elements identified in the selected cluster (31-bar)

can be adapted to the actual results, with the best damage result being obtained for the case where the maximum number of vibration modes ( 8 modes) was used. In this last case, the resultant damage for certain elements was negligible (these elements were actually undamaged). The results obtained from the non-clustering method with eight modes were also very different from the actual results. Fig. 5 compares the results obtained from the methods implementing the clustering technique and those obtained from non-clustering methods. Note that in [30], the final results from damage Scenario I were obtained by considering 10 modes as well as differences between the final damage values. Accordingly, some undamaged elements were represented as damaged elements where the assessed damage to Element 11 was approximately 0.28 .

As already mentioned, three damage scenarios were considered for this example. In the second and third scenarios, a similar process to Scenario I was followed. Fig. 6 shows the results obtained for the clusters selected from Damage Scenarios II and III. Then, as in Scenario I, the selected cluster in each scenario was identified and the optimization algorithm was run for that cluster. Fig. 7 displays the convergence trend in the optimization processes obtained for the selected clusters in scenarios II and III.

As observed in Fig. 7 and Fig. 8, the proposed method was an efficient method for detecting the location and severity of structural damage. This fact is demonstrated by comparing the results obtained from the proposed method with those obtained by Messina et al. [30]. For example, Scenario III shows that some of the actually undamaged elements were damaged. It determines a value of damage for Element 2 which is very different from the actual damage (equal to 0.06). The results were obtained in [30] by considering 10 modes for the structure, while in Fig. 8, the proposed algorithm managed to accurately detect the damaged elements by considering only three modes, while producing damage results that are exactly equal to the actual values [30]. Note that - in damage Scenarios II and III - the results were obtained without analyzing the clustering method. The results in such case were almost equal to those obtained from Scenario I.

\subsection{A 72-bar spatial truss}

To evaluate the performance of the proposed algorithm, a space truss with 72 elements was selected based on the assumptions in [31], as shown in Fig. 9. $E$ and $\rho$ used in all elements were assumed to be $6.98 \times 1010 \mathrm{~N} / \mathrm{m}^{2}$ and $2770 \mathrm{~kg} /$ $\mathrm{m}^{3}$, respectively. The cross-sectional area for all the elements was assumed to be $0.0025 \mathrm{~m}^{2}$. Four non-structural

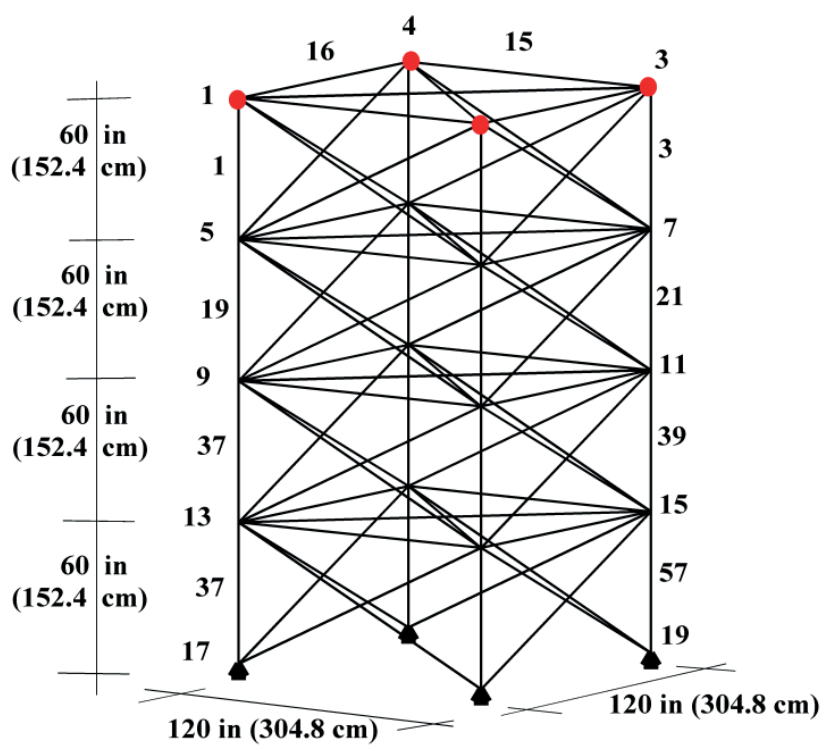

Fig. 9 Spatial truss with 72 elements 
masses of $2270 \mathrm{~kg}$ were applied to nodes 1 to 4 , respectively. In the damage location and severity detection process applied to the truss in [31], the first ten natural frequencies of the structure were used for obtaining the same dynamic characteristics. In the proposed method, however, only the first five modes were implemented. Two damage scenarios were considered for the space truss with 72 elements discussed in [31], as shown in Table 3.

The process used for detecting the damage location and severity applied to the space truss with 72 elements is similar to the one implemented for the 31-bar truss. Accordingly, the elements for each cluster were initially selected according to Table 4.

Next, the initial values for the damage obtained for the elements in each cluster were generally specified for each scenario (Fig. 10).

Ultimately, the selected cluster members were determined based on the results obtained from each scenario in accordance with Fig. 10, and the proposed method was applied to the selected cluster. Figs. 11 and 12 indicate the convergence trends and the numerical values of the damage

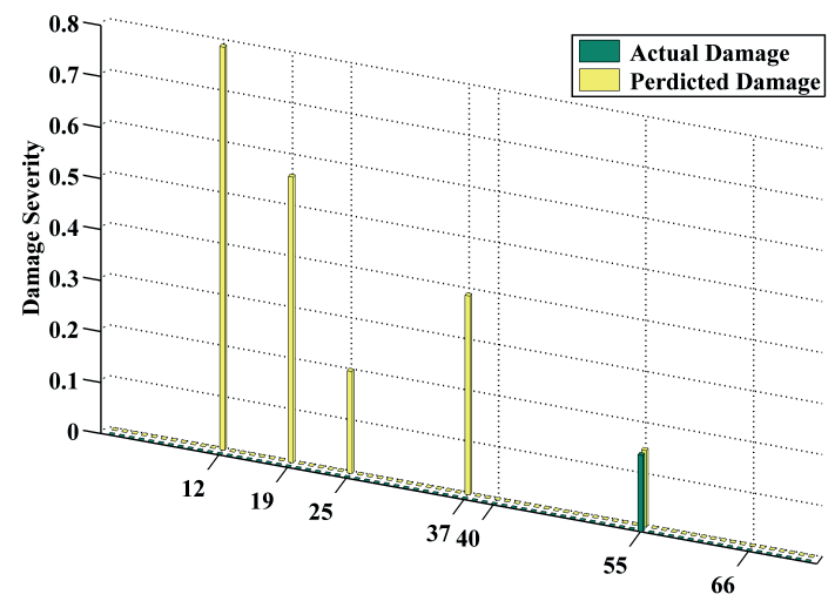

Scenario I

Fig. 10 Suspected damage elements based on clustering (72 bar)
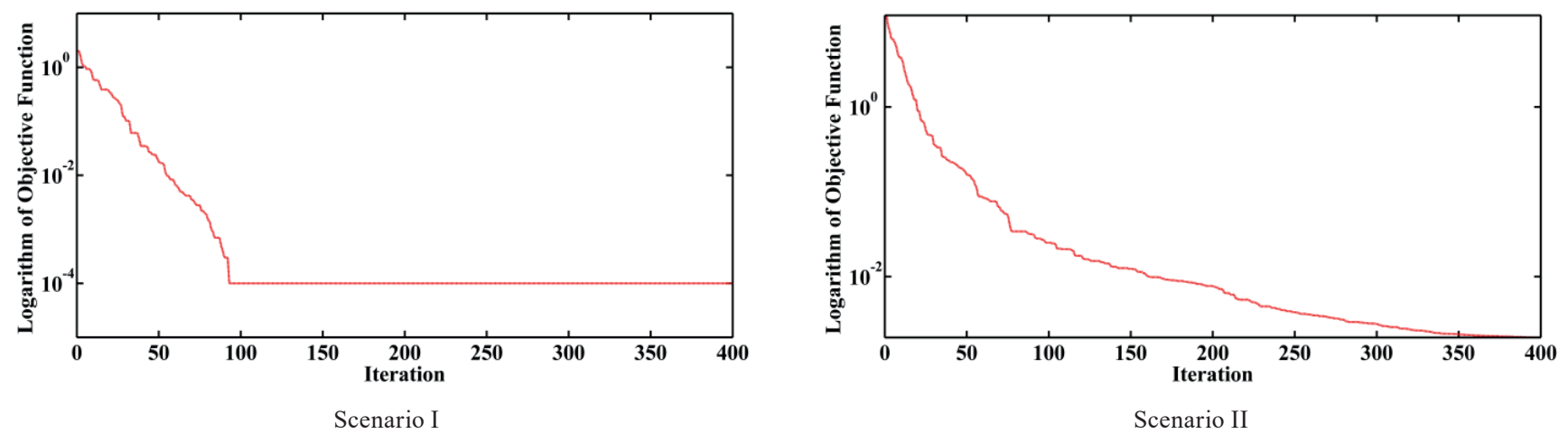

Fig. 11 Convergence of the optimization process to specify the location and severity of damage (72 bar)
Table 3 Three different damage scenarios induced in 72 bar truss

\begin{tabular}{|c|c|c|}
\hline Scenaric & Element $\mathrm{No}$ & Damage ratio \\
\hline I & 55 & 0.15 \\
\hline \multirow{2}{*}{ II } & 4 & 0.15 \\
\hline & 58 & 0.1 \\
\hline \multicolumn{3}{|c|}{ Table 4 Clustering of the truss elements (72-bar) } \\
\hline $\begin{array}{l}\text { Cluster } \\
\text { No. }\end{array}$ & $\begin{array}{c}\text { The members of cluster } \\
\text { Scenario I }\end{array}$ & $\begin{array}{c}\text { The members of cluster } \\
\text { Scenario II }\end{array}$ \\
\hline 1 & $21,22,25,26,29,30,31,33,37,40$ & $1,2,4,7,8,11,12,13,15,19,20,22$ \\
\hline 2 & $55,56,61,62,65,66$ & $25,26,29,30,31,33,37,38,40,43$ \\
\hline 3 & $3,4,7,8,11,12,13,15,19$ & $47,49,51,56,58,61,62,65,66$ \\
\hline
\end{tabular}

severity obtained from these scenarios. As observed in Fig. 12, the damage severity detection process applied to the second scenario correctly identified the damaged elements. However, the damage calculated for one element was different from the actual damage by a small margin of error, and the damage obtained for Element 1 was very slight. In both cases, the observed differences were negligible. In other words, due to the slight differences observed,

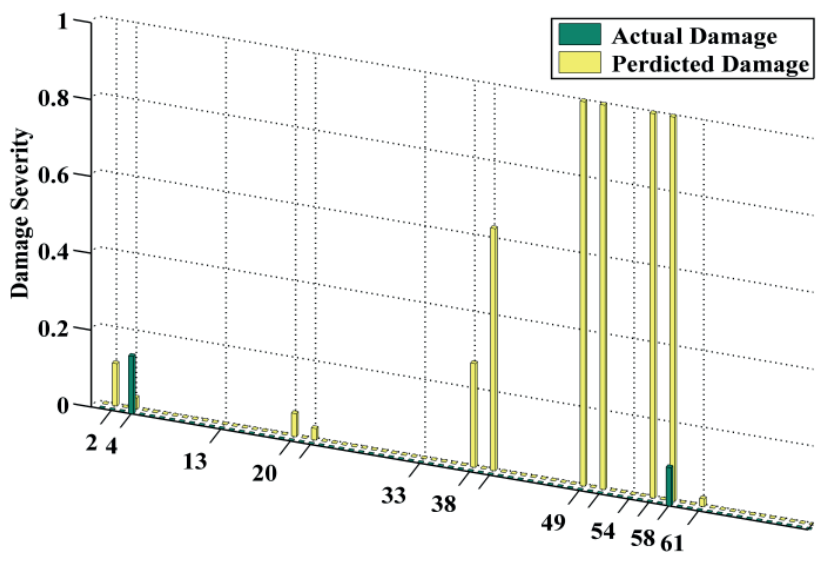

Scenario II 


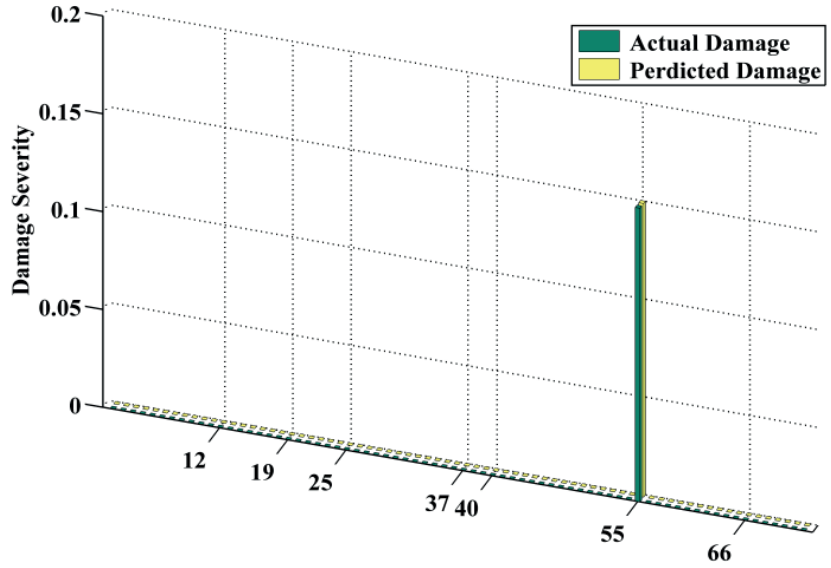

Scenario I

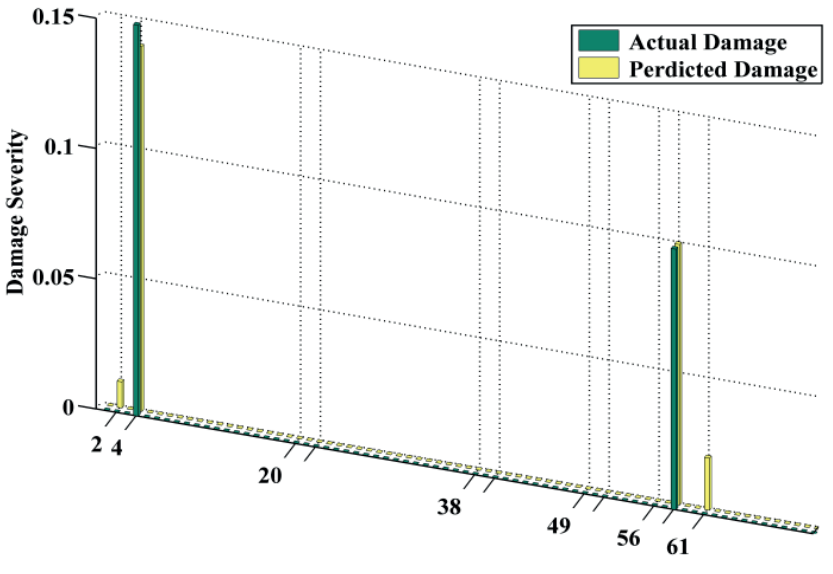

Scenario I

Fig. 12 The damage elements identified in the selected cluster ( 72 bar)

it was concluded that the obtained results were acceptable. The results obtained from Scenario I fully matched the actual results. Note that in [31], the numerical results for damage were not given, and therefore, no comparison could be made in this regard. The examination of the results obtained from the conventional methods (no clustering) in this example proved that the proposed method was a suitable damage detection method.

\subsection{A 47-bar planar truss}

The efficiency of the proposed method was tested - for the first time in this paper - by applying it to a tower with 47 elements (Fig. 13).

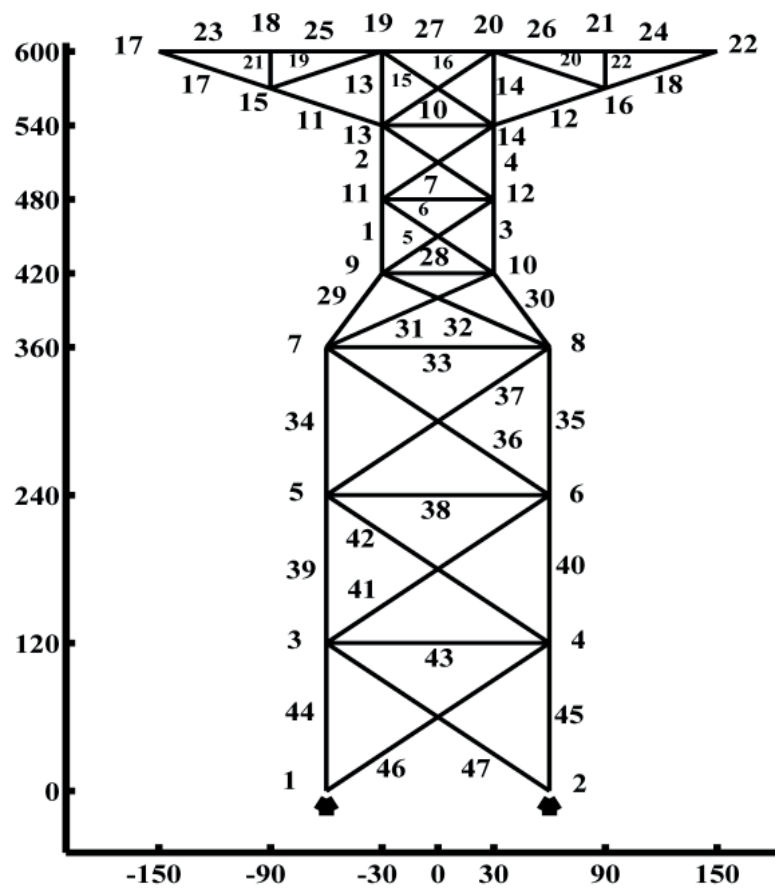

Fig. 13 A 47-bar planar truss structures
The above structure had 47 elements and 22 nodes. It was assumed that the first 10 natural frequencies of the structure were available (for determining the dynamic characteristics of the structure). For all the elements, $E$ and $\rho$ were assumed $206842.8 \mathrm{GPa}$ and $303.97 \mathrm{~kg} / \mathrm{m}^{3}$, respectively. The cross sectional area of all the elements was based on the values given in [32] for the optimum design. In this example, the efficiency of the proposed method was demonstrated through two scenarios, as shown in Table 5 (negligible damage was assumed in the elements).

Table 6 indicates the results obtained from the clustering method applied to each scenario.

Fig. 14 shows the damage values obtained for the structural elements in each scenario from the first step of the proposed method. Figs. 15 and 16 show the convergence trends and the numerical values of the damage severity obtained for the studied structure in each scenario.

\begin{tabular}{lcc}
\multicolumn{3}{c}{ Table 5 Three different damage scenarios induced in 47 bar truss } \\
\hline Scenario & Element No & Damage ratio \\
\hline \multirow{2}{*}{ I } & 7 & 0.05 \\
& 36 & 0.15 \\
II & 27 & 0.05 \\
& 42 & 0.05
\end{tabular}

Table 6 Clustering of the truss elements (47-bar)

\begin{tabular}{lcc}
\hline $\begin{array}{l}\text { Cluster } \\
\text { No. }\end{array}$ & $\begin{array}{c}\text { The members of cluster } \\
\text { Scenario I }\end{array}$ & $\begin{array}{c}\text { The members of cluster } \\
\text { Scenario II }\end{array}$ \\
\hline 1 & $17,18,19,20,22,23,24,25,26$, & $22,24,26,27,31,32,34,36$ \\
$27,28,31,33$ & $1,6,8,10,11,12$, \\
2 & $1,2,7,8,10,11,12$ & $13,15,18,20$ \\
3 & $36,37,41,42,43,44,45,46,47$ & $38,41,42,43,45,46,47$ \\
\hline
\end{tabular}




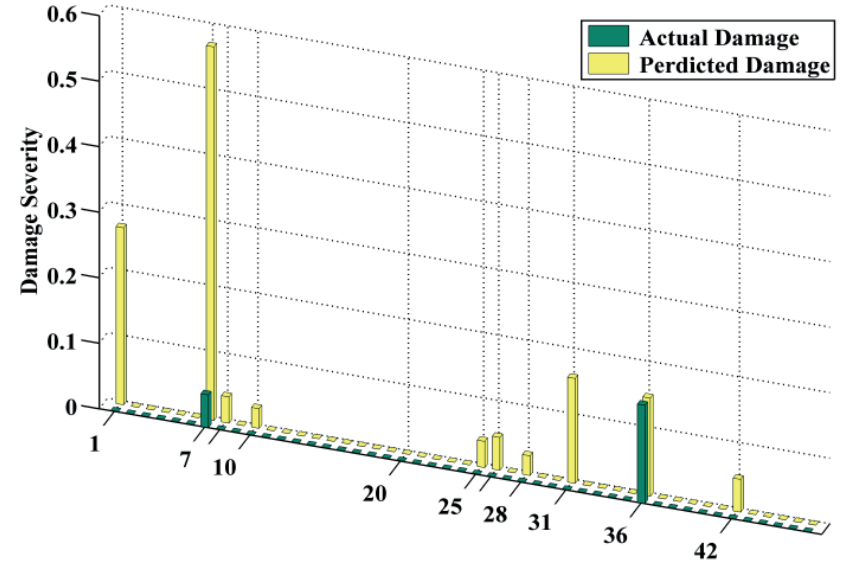

Scenario I

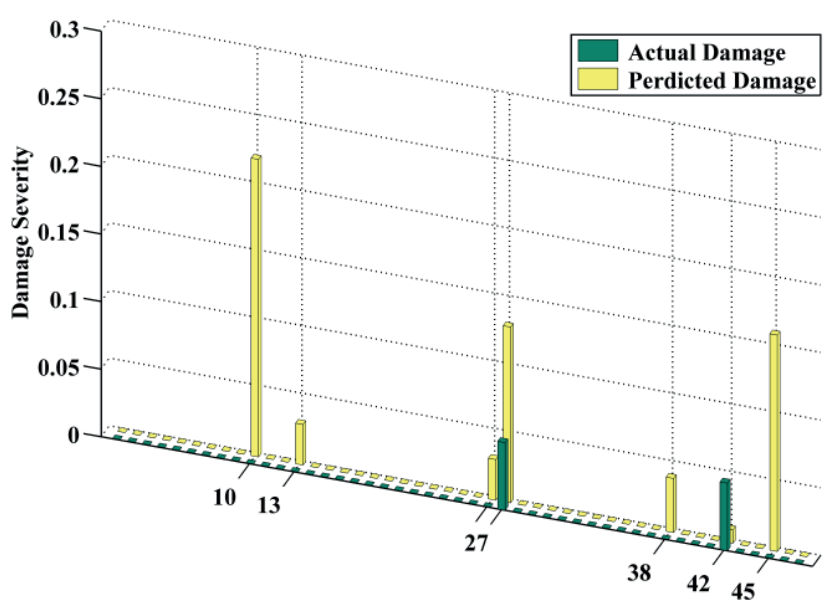

Scenario II

Fig. 14 Suspected damage elements based on clustering (47 bar)
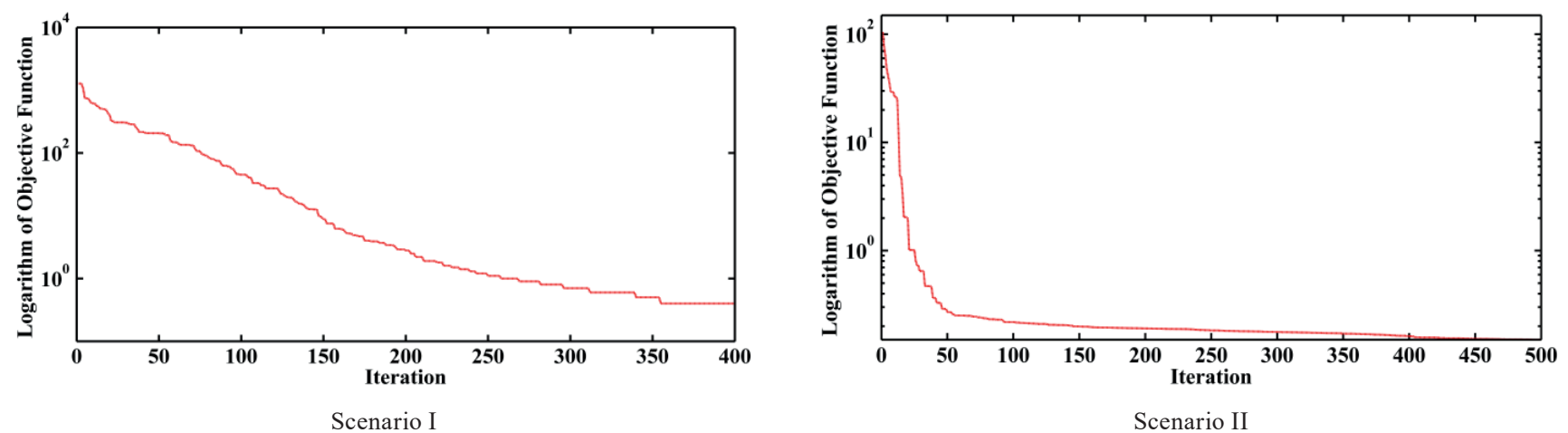

Fig. 15 Convergence of the optimization process to specify the location and severity of damage (47 bar)

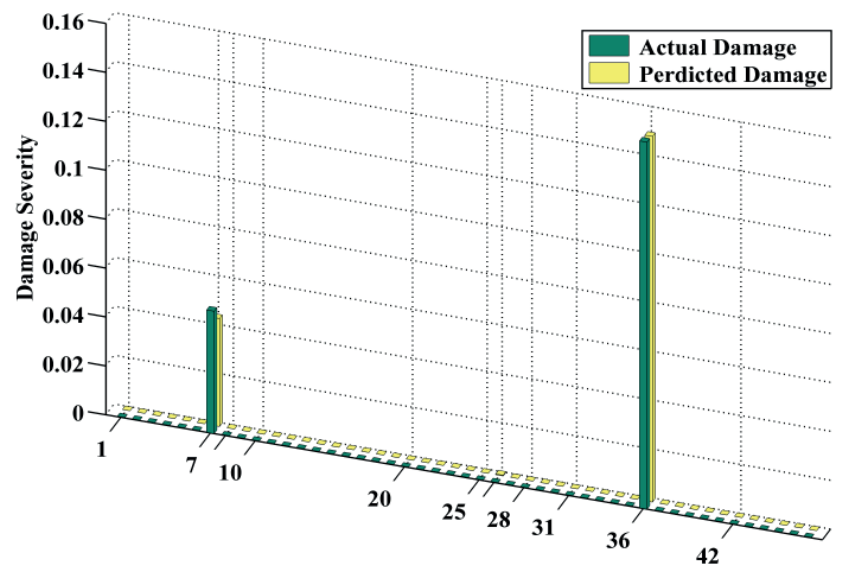

Scenario I

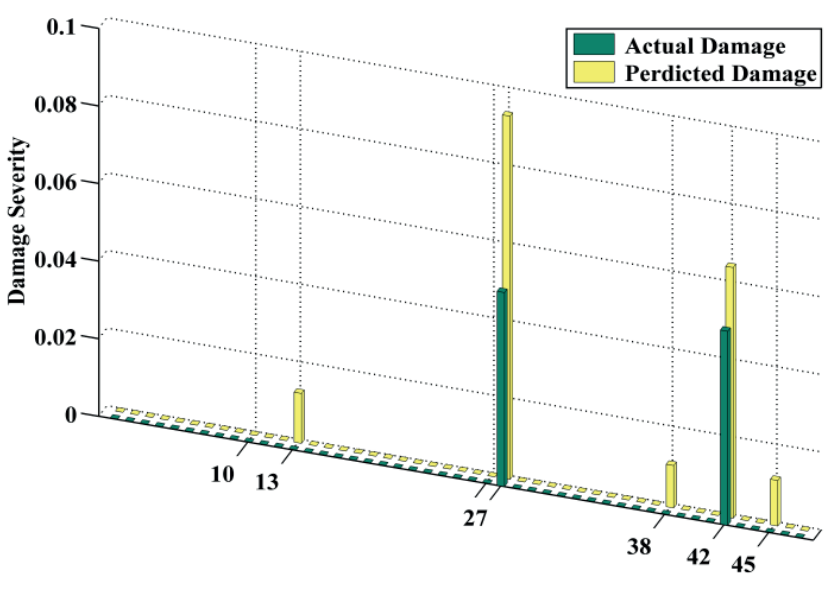

Scenario II

Fig. 16 The damage elements identified in the selected cluster (47 bar)

In this example, the efficiency of the proposed method was evaluated by assuming a slight damage in the elements. Due to the high structural stiffness of the elements, this value was slight and difficult to recognize by the algorithm. In other words, a reduction of 0.05 in the stiffness of one or two structural elements would have a very slight effect on the structural characteristics of the whole structure. According to the results, the damage location and severity values obtained from the proposed method were very slightly different from the damage extent values. 


\section{Conclusions}

A new method was proposed for detecting structural damage based on the modal strain energy and the idea of clustering structural elements through the k-means method combined with the $\mathrm{CBO}$ algorithm. By clustering the elements and using the limited data extracted from the structure itself, the location and severity of the structural damage can be detected with good accuracy. The idea of clustering structural elements in the optimization process would narrow down the search space while preventing local optimum entrapment. To monitor the structural health in the proposed method, the suspected damage elements were first selected by comparing the stresses developed in the undamaged and damaged elements. Then, the proposed clustering idea was employed to divide the suspected damage elements into several clusters. The $k$-means method was implemented for clustering the elements. This statistical method can be favorably used in large search spaces for classifying points with identical characteristics in specific groups. A damage detection criterion applicable to suspected damage elements was subsequently described by considering the variations of their modal strain energy. To this end, the strain energy equation was first expanded. Then, the total stiffness matrix of the damaged structure was transformed into the sum of the stiffness matrices of its individual members. During the matrix transformation process (which converted the total stiffness matrix into individual stiffness matrices for individual elements), unknown coefficients were incorporated into the equation for expressing the damage probability of individual elements. One characteristic of this method is that it uses only a limited number of frequencies for damage detection

\section{References}

[1] Doebling, S. W., Farrar, C. R., Prime, M. B. "A summary review of vibration-based damage identification methods", The Shock and Vibration Digest, 30(2), pp. 91-105, 1998. [online] Available at: https://pdfs.semanticscholar.org/1efa/222a89838ba52fd3846970 4bd83aa3b4cad8.pdf

[2] Doebling, S. W., Hemez, F. M., Peterson, L. D., Farhat, C. "Improved Damage Location Accuracy Using Strain Energy-Based Mode Selection Criteria", AIAA Journal, 35(4), pp. 693-699, 1993. https://doi.org/10.2514/2.159

[3] Bernal, D. "Load Vectors for Damage Localization", Journal of Engineering Mechanics, 128(1), pp. 7-14, 2002. https://doi.org/10.1061/(ASCE)0733-9399(2002)128:1(7)

[4] Zonta, D., Lanaro, A., Zanon, P. "A strain-flexibility-based approach to damage location", Key Engineering Materials, 245-246, pp. 87-94, 2003.

https://doi.org/10.4028/www.scientific.net/KEM.245-246.87 and does not require full knowledge of the damaged elements. Further, it can detect the slightest extent of damage ( $>5 \%$ ). These characteristics can be clearly observed in the given examples (Section 4.3). To solve the governing equation in the damage process, the meta-heuristic CBO algorithm was used to formulate the above equation as an unconstrained optimization problem. Accordingly, the energy equation was expanded for the elements in each cluster and solved accordingly through an iteration procedure. Upon completing each step of the algorithm in each cluster, the suspected damage elements were identified and grouped together in the form of a selected cluster. Ultimately, the energy equation was applied to the selected cluster (set) and solved to yield the location and severity of damage for each damaged element. Three examples (with different geometries and different numbers of elements) were selected from different references to check the performance of the proposed method. The results show that the proposed method is able to detect small damages $(>5 \%)$. However, due to the formulation of the problem at a very slight extent of damage, the optimization process for detecting damage would be somewhat difficult, resulting in identification of undamaged elements as slightly damaged elements. This is due to the number of the unknowns (i.e. the number of the elements) being considerably greater than that of the equations (i.e. the number of vibration modes), which would cause the lack of a unique solution for the governing equations. Nevertheless, the obtained results were deemed acceptable in view of the very slight differences found through the proposed method. Therefore, the proposed method is recommended for the detection of damage location and severity in structures.

[5] Pandey, A. K., Biswas, M. "Damage Detection in Structures Using Changes in Flexibility", Journal of Sound and Vibration, 169(1), pp. 3-17, 1994.

https://doi.org/10.1006/jsvi.1994.1002

[6] Sohn, H., Law, K. H. "Damage Diagnosis Using Experimental Ritz Vectors", Journal of Engineering Mechanics, 127(11), pp. 11841193, 2001. https://doi.org/10.1061/(ASCE)0733-9399(2001)127:11(1184)

[7] Shi, Z. Y., Law, S. S., Zhang, L. M. "Structural Damage Detection from Modal Strain Energy Change", Journal of Engineering Mechanics (ASCE), 126(12), pp. 1216-1223, 2000. https://doi.org/10.1061/(ASCE)0733-9399(2000)126:12(1216)

[8] Guan, H., Karbhari, V. M. "Improved damage detection method based on Element Modal Strain Damage Index using sparse measurement", Journal of Sound and Vibration, 309(3-5), pp. 465-494, 2008. https://doi.org/10.1016/j.jsv.2007.07.060 
[9] Yang, Q. W. "A numerical technique for structural damage detection", Applied Mathematics and Computation, 215(7), pp. 27752780, 2009.

https://doi.org/10.1016/j.amc.2009.08.039

[10] Chou, J.-H., Ghaboussi, J. "Genetic algorithm in structural damage detection", Computer and Structures, 79(14), pp. 1335-1353, 2001. https://doi.org/10.1016/S0045-7949(01)00027-X

[11] Perera, R., Fang, S.-E., Ruiz, A. "Application of particle swarm optimization and genetic algorithm to multiobjective damage identification inverse problems with modeling errors", Meccanica, 45, pp. 723-734, 2010.

https://doi.org/10.1007/s11012-009-9264-5

[12] Wang, Y., Liu, J., Shi, F., Xiao, J. "A Method to Identify Damage of Roof Truss under Static Load Using Genetic Algorithm", In: Proceedings of the International Conference on Artificial Intelligence and Computational Intelligence (AICI), Sanya, China, 2010, pp. 9-15.

https://doi.org/10.1007/978-3-642-16527-6_2

[13] Seyedpoor, S. M. "A two stage method for structural damage detection using a modal strain energy based index and particle swarm optimization", Journal of Non-Linear Mechanics, 47(1), pp. 1-8, 2012.

https://doi.org/10.1016/j.ijnonlinmec.2011.07.011

[14] Kaveh, A., Maniat, M. "Damage detection based on MCSS and PSO using modal data", Smart Structures and Systems, 15(5), pp. 1253$1270,2015$.

https://doi.org/10.12989/sss.2015.15.5.1253

[15] Kaveh, A., Motie Share, M. A., Moslehi, M. "Magnetic charged system search: a new meta-heuristic algorithm for optimization", Acta Mechanica, 224, pp. 85-107, 2013.

https://doi.org/10.1007/s00707-012-0745-6

[16] Kaveh, A., Mahdavi, V. R. "Damage identification of truss structure using $\mathrm{CBO}$ and ECBO algorithms", Asian Journal of Civil Engineering, 17(1), pp. 75-89, 2016. [online] Available at: https:// ajce.bhrc.ac.ir/Portals/25/PropertyAgent/2905/Files/6455/75.pdf

[17] Kaveh, A., Zolghadr, A. "Guided Modal Strain Energy-Based Approach for Structural Damage Identification Using Tug-ofWar Optimization Algorithm", Journal of Computing in Civil Engineering, 31(4), 2017.

https://doi.org/10.1061/(ASCE)CP.1943-5487.0000665

[18] Kaveh, A., Dadras, A. "Structural damage identification using an enhanced thermal exchange optimization algorithm", Engineering Optimization, 50(3), pp. 430-451, 2017.

https://doi.org/10.1080/0305215X.2017.1318872

[19] Kaveh, A., Zolghadr, A. "Cyclical Parthenogenesis Algorithm for guided modal strain energy based structural damage detection", Applied Soft Computing, 57, pp. 250-264, 2017. https://doi.org/10.1016/j.asoc.2017.04.010

[20] Kaveh, A., Zolghadr, A "Cyclical parthenogenesis algorithm: A new meta-heuristic algorithm", Asian Journal of Civil Engineering, 18(5), pp. 673-701, 2017.
[21] Yang, Q. W., Liu, J. K. "Damage identification by the eigenparameter decomposition of structural flexibility change", International Journal for Numerical Methods in Engineering, 78(4), pp. 444-459, 2009.

https://doi.org/10.1002/nme.2494

[22] Torkzadeh, P., Goodarzi, Y., Salajegheh, E. "A two-stage damage detection method for large-scale structure by kinetic and modal strain energies using heuristic particle swarm optimization", International Journal of Optimization in Civil Engineering, 3(3), pp. 465-482, 2013. [online] Available at: http://www.iust.ac.ir/ijoce/ article-1-144-en.html

[23] Wang, X., Hu, N., Fukunaga, H., Yao, Z. H. "Structural damage identification using static test data and changes in frequencies", Engineering Structures, 23(6), pp. 610-621, 2001. https://doi.org/10.1016/S0141-0296(00)00086-9

[24] Bakhtiari-Nejad, F., Rahai, A., Esfandiari, A. "A structural damage detection method using static noisy data", Engineering Structures, 27(12), pp. 1784-1793, 2005. https://doi.org/10.1016/j.engstruct.2005.04.019

[25] Jain, A. K., Dubes, R. C. "Algorithms for clustering data", PrenticeHall, Upper Saddle River, NJ, USA, 1988.

[26] Kaufman, L., Rousseeuw, P. J. "Finding Groups in Data: An Introduction to Cluster Analysis", John Wiley \& Sons, Hoboken, NJ, USA, 1990. https://doi.org/10.1002/9780470316801

[27] Likas, A., Vlassis, N., Verbeek, J. J. "The global k-means clustering algorithm", Pattern Recognition, 36(2), pp. 451-461, 2003. https://doi.org/10.1016/S0031-3203(02)00060-2

[28] Kaveh, A., Mahdavi, V. R. "Colliding Bodies Optimization method for optimum discrete design of truss structures", Computers \& Structures, 139, pp. 43-53, 2014. https://doi.org/10.1016/j.compstruc.2014.04.006

[29] Kaveh, A., Ilchi Ghazaan, M. "A comparative study of CBO and ECBO for optimal design of skeletal structures", Computers and Structures, 153, pp. 137-147, 2015. https://doi.org/10.1016/j.compstruc.2015.02.028

[30] Messina, A., Williams, E. J., Contursi, T. "Structural damage detection by a sensitivity and statistical-based method", Journal of Sound and Vibration, 216(5), pp. 791-808, 1998. https://doi.org/10.1006/jsvi.1998.1728

[31] Kaveh, A., Zolghadr, A. "An improved CSS for damage detection of truss structures using changes in natural frequencies and mode shapes", Advances in Engineering Software, 80, pp. 93-100, 2015. https://doi.org/10.1016/j.advengsoft.2014.09.010

[32] Kalatjari, V. R., Talebpour, M. H. "An Improved Ant Colony Algorithm for the Optimization of Skeletal Structures by the Proposed Sampling Search Space Method", Periodica Polytechnica Civil Engineering, 61(2), pp. 232-243, 2017. https://doi.org/10.3311/PPci.9153 